\title{
Reply to Letter to Editor regarding "Economic impact of intraoperative parathyroid hormone assay in primary hyperparathyroidism?"
}

Dear Editor,

We read with interest the comments of de la Plaza Llamas et al. on our published manuscript "Cost-benefit analysis of the intraoperative parathyroid hormone assay in primary hyperparathyroidism."

Our analysis was based exclusively on the cost of parathyroid hormone assay and the operating room costs because they were objectively determined measures. The length of hospital stay was not considered because all patients stayed 1 night in the hospital, with the exception made for some patients of group A, who stayed more than 1 night due to long operating room time. We never affirmed that one of the benefits of using intraoperative intact parathyroid hormone (IOPTH) is that it shortens the length of stay in the hospital. Re-reading our article, we noticed that our conclusions can lead to a misunderstanding: when we state "this approach can decrease costs..." we meant "our approach," without IOPTH assay.

We think that the case of reoperation in group A reinforces our thesis about the overuse of IOPTH; nevertheless, we performed IOPTH and if we did not find the adenoma, we would have obtained the same result even without IOPTH essay, this is the reason why we think this case influenced the costs of group A.

We explained why delayed IOPTH was performed in group B1: it was our control group: "the B1 group was considered our control group because the results of PTH intraoperative sampling could not change the surgeon's strategy during the operation and it was used to better understand if IOPTH would be helpful in guiding the surgeon's intraoperative decisions."

The IOPTH was performed in all patients of group A, even in those 29 patients who underwent an associated thyroidectomy because at the beginning of our experience we planned to perform IOPTH to all patients. In addition, group B includes patients who underwent a thyroidectomy and a bilateral exploration. We think that the kind of intervention does not impact the diagnostic accuracy of IOPTH.

We do not perform minimally invasive video-assisted parathyroidectomy (MIVAP) with the patients under local anesthesia, in our experience, MIVAP is not longer than conventional or minimally invasive parathyroidectomy. Hence, MIVAP did not negatively influence operative time.

This is an observational-retrospective study. The slight dishomogeneity of the 2 groups is intrinsic in the study design but it did not affect costs because we considered homogeneous and objective parameters for the cost analysis.

We thank our colleagues for their comments from "the use of IOPTH produced false negative in twelve....." up to the end of the letter; we appreciated them but we do not think they are related to the primary purpose of our study: cost benefits.

Benedetta Badii MD, Fabio Staderini MD, Caterina Foppa MD, Lorenzo Tofani PhD, Ileana Skalamera MD, Giulia Fiorenza MD, Etleva Qirici MD, Fabio Cianchi MD, Giuliano Perigli MD University of Florence

Correspondence Fabio Staderini, Department of Medical and Surgical Critical Care, Medical School, University of Florence, Florence, Italy. Email: staderini.fabio@gmail.com 